\title{
La traducción del espacio novelesco de Fernando Contreras Castro y su transferencia a la realidad alemana (la estética de la recepción y traducción como factores determinantes en la oposición «literatura universalista - literatura localista») ${ }^{1}$
}

Juan Antonio Albaladejo 2

Universidad de Alicante, España

\section{RESUMEN}

Se señala la pertinencia, cultural e histórica de las letras costarricenses, y el escaso conocimiento en el entorno europeo. A partir del análisis de la traducción al alemán de la novela Los Peor, de Fernando Contreras, se estudian dos aspectos: la localidad esencial en la discursividad literaria del texto original, y las operaciones respectivas que desde ese mismo punto de vista se ejerce en la traducción. Se comenta como rasgo destacado el efecto de extrañamiento que emite la obra, favorable como expectativa estética en el lector europeo; ello propicia interés literario, traductológico y editorial.

Ponencia presentada en el I Congreso Internacional de Lingiǘstica Aplicada, llevado a cabo en octubre de 2007, en el Campus Omar Dengo, de la Universidad Nacional de Costa Rica.

2 Correo electrónico: ja.albaladejo@ua.es 


\begin{abstract}
Reference is made to the cultural and historical relevance of Costa Rican literature and the fact that it is not well known in Europe. With the analysis of the German translation of Los Peor by Fernando Contreras, two aspects are addressed: the essential place of the literary discourse of the original text and the respective operations which take place from that same perspective in the translation. One outstanding feature mentioned is the effect of estrangement that this novel conveys; this is considered positive by the European reader and promotes interest from the perspective of literature, translation and publishers.
\end{abstract}

Palabras clave: traducción, literatura costarricense contemporánea, narrativa costarricense. teoría de la recepción. identidad cultural.

Keywords: translation, Costa Rican contemporary literature, Costa Rican narrative, theory of reception, cultural identity.

Cuando los que somos ajenos a la realidad costarricense pensamos en Costa Rica nos asaltan, en primer lugar, imágenes de playas paradisíacas, colinas frondosas y, en general, una vegetación exuberante y exótica. Al fin y al cabo, los clichés turísticos no sólo funcionan sino que se están imponiendo cada vez más en el mundo actual. En un segundo momento, sin embargo, y por ignorancia de aquel que ve el resto del mundo con anteojos europeos, surgen en nosotros las típicas asociaciones centroamericanas, pues las escasas noticias que hoy en día llegan de allí inducen a identificar a todos los países de la región con una única realidad. Por un lado, aparecen desde un lejano rincón de nuestra memoria recuerdos de conflictos sociales, duras luchas políticas, guerras civiles, golpes militares, regímenes totalitarios y las luchas guerrilleras. Por otra parte, las únicas noticias que, al parecer, logran en los últimos tiempos cruzar «el Gran Charco» son las de las catástrofes naturales tales como los huracanes y los terremotos. Estos son algunos de los prejuicios que determinan en buena medida el primer encuentro con Costa Rica ${ }^{3}$.

3 W. Mackenbach, «Vorwort», W. Mackenbach, (ed.), Papayas und Bananen. Erotische und andere Erzählıngen aus Zentralamerika. (Frankfurt am Main: Brandes \& Apsel, 2002), 7: «Lange Zeit 
Si y a resulta poco conocida la realidad cotidiana costarricense al otro lado del Atlántico, ¿qué decir de su literatura? Entre las asociaciones habituales que se establecen con este pequeño país centroamericano - tal y como ya se ha indicado - no figura la identificación con las bellas letras. Pocos europeos serían capaz de nombrar algún autor costarricense, ya sea contemporáneo ya sea del pasado. A diferencia de lo que ocurre con muchos otros países que han participado del gran auge de la literatura hispanoamericana a partir de los años sesenta del siglo xx, Costa Rica aún no ha producido ninguna figura de relieve internacional hasta el momento. El hecho de haberse quedado al margen de la gran evolución que ha experimentado la literatura del sur del continente americano en las últimas décadas explica la práctica ausencia de huellas de las letras costarricenses en el mundo. En el tablón de anuncios del nuevo mundo globalizado, donde uno puede dar con las cosas más inverosímiles, extravagantes y raras, buscará en vano información detallada sobre lo que se ha escrito y lo que se está escribiendo en Costa Rica en la actualidad. El asiduo usuario del buscador monopolista Google permanece incrédulo ante una pantalla sospechosamente escasa de resultados. Pero por más que se insiste, esa escasez no se corrige y llega el momento en el que hay que concluir que efectivamente no hay apenas datos que se puedan recopilar.

El investigador, esencialmente arrastrado por el ímpetu de un compañero que pretende abrir nuevas líneas de trabajo y decide elegir el tema de la literatura costarricense y su recepción en otras lenguas, se encuentra rápidamente con un problema básico y muy sorprendente: las dificultades de acceder a los textos originales. A

war die europäische Wahmehmung Zentralamerikas von den politischen Ereignissen in der Region bestimmt. Auch das Interesse an der zentralamerikanischen Literatur war vor allem politisch geprägt und verband sich mit den hiesigen Hof fnungen auf eine Verwirklichung politischer Utopien unter dortigen, exotischen Bedingungen. Inzwischen ist die politische Auf merksamkeit für Zentralamerika weitgehend erloschen - in den Schlagzeilen taucht die Region nur noch bei Naturkatastrophen auf-, und auch das Interesse an der Literatur der Region ist geschwunden». 
diferencia de lo que, en un principio, se podría pensar, los textos españoles no están disponibles en España, ni en las librerías ni en las bibliotecas. Lamentablemente hay que reconocer que la biblioteca de nuestra Universidad de Alicante cuenta con un único libro de un autor costarricense: se trata de la novela Mamita Yunai de Carlos Fallas.

Ante este panorama desolador en la Península Ibérica, nuestro interés investigador se centró en descubrir la suerte que ha corrido la literatura de Costa Rica en otros países. El descubrimiento, nuevamente sorprendente, de que en Alemania se había editado en 2002 Der Mönch, das Kind und die Stadt, una traducción al alemán de la novela Los Peor del costarricense Fernando Contreras Castro, impulsó la búsqueda del texto original que finalmente se localizó en Estados Unidos.

El texto costarricense presenta, a primera vista, elementos de extrañamiento y algunos problemas de comprensión para el lector español que se deben a los contrastes, sobre todo, léxicos pero también fraseológicos y sintácticos respecto de los usos lingüísticos peninsulares. Así, por ejemplo, aparecen en la novela palabras como gallopinto, chunches, jareta o polvo de gallo que provocan, en un principio, un fruncir del ceño en el lector español no familiarizado con los «tiquismos» o costarriqueñismos. A pesar de la existencia de contrastes intralingüísticos estos no plantean serias dificultades de comprensión que pudieran imposibilitar la recepción global del texto. Por una parte, el contexto permite a menudo deducir los significados desconocidos y, por otra parte, la novela trasciende los estrechos límites geográficos en los que se desarrolla la trama, aunque se establezca una íntima relación entre la acción y el espacio físico en el que se mueven los personajes.

Como corresponde a una novela «de bajos fondos» —así la califica Tatiana Lobo en la contraportada del libro-el lenguaje refleja cierto grado de coloquialismo, sobre todo en el discurso directo de los personajes, aunque incluso se percibe en el discurso del narrador, algo que sí resulta más chocante. Respecto del elemento coloquial se 
observa en la traducción una tendencia niveladora que, aunque no llegue al grado máximo de la neutralización, si refleja un carácter atenuante. Tratándose como se trata de una traducción comercial y no filológica, pues carece por completo de aparato crítico, no es de extrañar que el traductor evite elementos que puedan provocar un rechazo, en primer lugar, del editor y en último término del lector. Las soluciones que emplea el traductor en el siguiente pasaje quizá se deban precisamente al intento de evitar herir el sentido estético de la mayoría de los lectores:

-Usté cré que luhago porque me gusta, ...iputa viejo más tonto!, pa comer, pa qué va a ser ${ }^{4}$.

„Sie meinen wohl, ich mach' das, weil's mir Spaß macht? Schaut euch mal den verrückten Alten an! Um was zu beißen zu haben, natürlich, weshalb denn sonst?"'s

La solución del traductor de emplear coloquialismos generalizados en la lengua y que carecen de cualquier connotación diatópica (aquí la habitual síncopa de la /e/átona del verbo en primera persona, la aféresis de la/e/ del pronombre personal neutro con la consiguiente contracción así como un registro léxico coloquial) produce sólo ligeros contrastes respecto de la lengua estándar. Esta estrategia del traductor podría deberse a la intención de evitar la evocación de paralelismos no deseados con lenguajes de carácter local y/o social de la lengua meta que pudieran marcar el texto en un sentido no intencionado por el autor costarricense. Bien es verdad que en el texto original los personajes que emplean un lenguaje claramente coloquial sí están marcados diastráticamente, con lo que el traductor podría haber

Fernando Contreras Castro, Los Peor (San José: Ediciones Farben, 1995) 179.

Fernando Contreras Castro, Der Mönch, das Kind und die Stadt (Augsburg: Maroverlag, 2002) 150. 
optado por soluciones más pronunciadas y más caracterizadoras en cuanto a la extracción social. Sea como fuere, el traductor opta por recrear los contrastes lingüísticos que presenta el texto original, aunque sin duda no llega a reproducir el mismo grado de contraste.

En términos generales, se percibe una tendencia de acercamiento al lector en la traducción, eligiendo por tanto el procedimiento más generalizado hoy en día que consiste en producir un texto meta que dé la sensación de ser un original ${ }^{6}$. Sin embargo, el traductor recurre al mismo tiempo a múltiples préstamos e incluso calca estructuras del español que contribuyen a producir el ef ecto contrario. Por ejemplo, la expresión "conocer al dedillo» ${ }^{7}$ se convierte en wie ihre eigene Handfläche kennen lernen ${ }^{8}$, o sea literalmente «conocer como la propia palma de la mano», expresión que no se usa en alemán aunque para el lector resulte transparente. En lo léxico se puede citar el caso de los «muros de adobes» ${ }^{9}$, en alemán Wände aus Adobeziegeln ${ }^{10}$, donde el traductor presta el término «adobe» y forma una palabra híbrida no habitual y probablemente opaca para el lector medio. En realidad, la correspondencia alemana sería Lehmziegel.

La problemática que este proceder implica ya la resaltaba Schleiermacher a principios del siglo xix en Sobre los diferentes métodos de traducir ${ }^{11}$. Son pocos los traductores que pueden resistir la

$6 \quad$ Lawrence Venuti, The Translator's Invisibility. A History of Translation (Londres/Nueva York: Routledge, 1995): «A translated text, whether prose or poetry, fiction or nonfiction, is judged acceptable by most publishers, reviewers, and readers when it reads fluently, when the absence of any linguistic or stylistic peculiarities makes it seem transparent, giving the appearance that it reflects the foreign writer's personality or intention or the essential meaning of the foreign text - the appearance, in other words, that the translation is not in fact a translation, but the original», 1 .

7 Contreras Castro, Los Peor, 48.

8 Contreras Castro, Der Mönch..., 38.

9 Contreras Castro, Der Mönch..., 69.

10 Contreras Castro, Der Mönch..., 56.

11 Friedrich Schleiermacher, Sobre los diferentes métodos de traducir (Madrid: Gredos, 2000): «O bien el traductor deja al escritor lo más tranquilo posible y hace que el lector vaya a su encuentro, 0 bien deja lo más tranquilo posible al lector y hace que vaya a su encuentro el escritor. Ambos son tan por completo dif erentes, que uno de ellos tiene que ser seguido con el mayor rigor, pues cualquier mezcla produce necesariamente un resultado muy insatisfactorio, y es de temer que el encuentro de escritor y lector falle del todo", 47. 
crítica a la que se enfrenta aquel que trata de mover al lector hacia el escritor y no a la inversa, es decir la crítica de no dominar bien la propia lengua materna. Schleiermacher formulaba así esta cuestión:

¿Quién admitirá de buena gana que le consideren torpe, mientras se esfuerza por conservar frente a la lengua extraña toda la proximidad que tolera la propia, y que se le censure como a los padres que entregan hijos a los volatineros, porque, en vez de ejercitar a su lengua materna en una gimnasia apropiada, trata de acostumbrarla a contorsiones extrañas y antinaturales? ${ }^{12}$

En lo literario, se percibe una novela que parece conectar con la tradición del realismo mágico el cual, en buena medida, ha contribuido a situar la literatura hispanoamericana dentro del canon literario universal. En su Historia de la Literatura Hispanoamericana, Giuseppe Bellini define el realismo mágico como «[...] la búsqueda de la realidad propia a través de la naturaleza, el mito y la historia, para afirmar el sello de la originalidad y de la unidad americana en el mundo» ${ }^{13}$. Además afirma que «[...] en el realismo mágico conviven numerosas características del regionalismo, del neorrealismo o de la novela de protesta» ${ }^{14}$. En Los Peor se observan estas influencias que se cristalizan en el concepto de realismo mágico pero con una notable diferencia: el elemento mitológico- histórico que recorre la novela no muestra un carácter esencialmente local-regional sino universal. Las alusiones a la mitología e historia greco-latina son constantes, sobre todo a partir de la identificación del niño deforme, uno de los personajes centrales de la novela, con la figura mitológica de Polifemo.

Sin desligarse de sus raíces, el texto trasciende lo meramente local y aspira a una proyección más allá de los límites del propio mundo de experiencias, algo que queda patente en las constantes

12 Schleiermacher, 69.

13 G. Bellini, Historia de la literatura hispanoamericana (Madrid: Castalia, 1985), 527.

14 Bellini, 527. 
alusiones a la mitología e historia que forman parte del patrimonio universal de la humanidad. No faltan las referencias a los problemas y miserias del propio medio tales como la urbanización deshumanizada, los atentados contra el medio ambiente y sus consecuencias para el ser humano o la violencia producto de la desesperación de los grupos sociales más desfavorecidos. Los elementos de denuncia abarcan un amplio espectro que incluye la corrupción política, los abusos policiales, el tráfico de órganos, las injusticias sociales y la contaminación con productos agroquímicos.

Contreras configura un texto en el que esos temas van incorporándose en torno a la historia nuclear de un niño que nace en un prostíbulo de San José con un solo ojo, probablemente por la exposición a los productos agroquímicos que sufrió su madre. Jerónimo Peor, un antiguo monje algo extravagante, hermano de la cocinera del prostíbulo, se hace cargo de la educación del pequeño, le pone el nombre de Polifemo —en alusión al célebre cíclope de la Odisea-e indica que es un «signo de nuestros tiempos». Durante años Jerónimo instruye al niño, recluido en el prostíbulo, en los saberes que el monje ha adquirido de los libros antiguos que leyó en su época monacal. Tras años de confinamiento, Polifemo finalmente descubre el mundo exterior y se convierte en un niño más, que a diario recorre las calles josefinas. Atraído por este mundo agitado y lleno de vida, Polifemo se va alejando más y más de su tutor. Pero justo cuando Jerónimo cree haber perdido a su pupilo éste cae enfermo como consecuencia de su deformidad y lo recupera gracias a la metamorfosis que experimenta el niño por la que se convierte en un limonero.

Las alusiones intertextuales que emplea el autor son permanentes y sirven, a menudo, como elementos de comparación e ilustración y le permiten fundir, de forma verosímil, lo real y lo mitológico. En su recensión en la Frankfurter Allgemeine Zeitung ${ }^{15}$, Klara Obermüller

15 K. Obermüller, «Der Knabe im Limonenbäumchen: Fernando Contreras hat für alles zwei Erklärungen », Frankfurter Allgemeine Zeitung (27-05-2003). 
indica el carácter abierto del libro pues, según ella, el autor no deja claro si el niño ha de interpretarse como una reminiscencia mitológica o como síntoma de un mundo enfermo. Lo real y lo mitológico entran constantemente en contacto y se mezclan, esencialmente a través de la figura del antiguo monje al que todos toman por loco. Los espacios vacíos ${ }^{16}$, que el autor establece intencionadamente en su texto, hacen que la labor de recreación que tiene que llevar a cabo el lector sea más importante.

El espacio desempeña una función esencial en la novela y se convierte en elemento central del texto, algo que se refleja en las múltiples descripciones de calles, edificios, monumentos y lugares de San José. Junto al niño y al monje, la ciudad es el verdadero protagonista de la novela. La traducción alemana capta bien este hecho y lo pone de relieve a través del título que recoge a los tres auténticos protagonistas de la historia: El niño, el monje y la ciudad. El espacio se desarrolla en diferentes niveles: por una parte está el espacio físico real que se mezcla, a través de los recuerdos del amigo ciego de Jerónimo, con el espacio del pasado de los años treinta del siglo xx. Éste se convierte en un espacio imaginado en la mente del monje que al cerrar sus ojos se traslada al San José de aquella época. El espacio que vive y describe el ciego Don Félix contrasta fuertemente con el lugar físico en el que se mueven los demás personajes. Los comentarios negativos sobre la ciudad actual de San José son constantes y proceden tanto de los personajes como del narrador de la novela.

El único que es capaz de entrar en el mundo del anciano invidente es Jerónimo: «Consuelo, he conocido una ciudad que sólo se puede ver con los ojos cerrados» ${ }^{17}$. Cuando cierra sus ojos se traslada

16 Concepto central de la teoría de la recepción literaria desarrollada por Jauss e Iser. La indeterminación que resulta de los espacios vacíos que ha dejado el autor en el texto contribuye a la poliinterpretabilidad de la obra literaria. Véase Luis A. Acosta, El lector y la obra (Madrid: Gredos, 1989): «[... ] es posible observar en el texto una serie de espacios vacios que tienen que ser llenados por el lector a fin de poder realizar la experiencia estética. El sentido que la estructura del texto pueda encerrar, se fija a lo largo del acto personal de lectura, de forma que el lector, mediante este acto, interviene en la constitución de la obra artística, una de cuyas peculiaridades básicas es la polifuncionalidad", 82.

17 Contreras Castro, Los Peor, 62. 
a esa otra ciudad amable y humana que aún conserva un carácter auténtico y diferenciado que mucho contrasta con la que se ve con ojos abiertos:

Esa tarde lo llevaron sobre la Calle 9 hasta la Iglesia de La Soledad, el mismo edificio que Jerónimo había visto tantas veces a ojos abiertos, pero imposible de identificar con la linda iglesia de antaño, desde que el mal gusto clerical había dado en pintarla con colores pastel como de torta de quinceañera con los que venían arruinando las iglesias del país. Jerónimo había pasado ya muchas veces por ahí, pero con los ojos abiertos, lo cual hacía una sensible diferencia porque la calle a ojos abiertos era el Paseo de los Estudiantes, con una gasolinera al inicio y una presa insufrible de autos [...]. Pero a ojos cerrados, la Calle 9 era una apacible callecita con un empedrado parejo, rodeada de solares y parques con barandas llenos de árboles y plantas ornamentales $[\ldots]^{18}$.

Cuanto más se adentra Jerónimo en esa otra ciudad más se convence de que se trata de dos ciudades distintas, no sólo de dos estados diferentes de un mismo espacio separado por el tiempo: «[...] Jerónimo le expuso con tanta claridad cómo se trataba de dos ciudades absolutamente diferentes y más bien se mostró muy extrañado de que ella creyera que era la misma, viendo que aquella que se veía a ojos abiertos era especialmente fea y sin historia» ${ }^{19}$. La ausencia de pátina, pues «San José había renunciado a su pasado» ${ }^{20}$, hace que Jerónimo no asocie la nueva ciudad con aquella que ve con los ojos abiertos. Además, a diferencia de lo que ocurre con las personas, Jerónimo afirma que las ciudades rejuvenecen con el paso del tiempo volviéndose cada vez más jóvenes ${ }^{21}$.

\footnotetext{
18 Contreras Castro, Los Peor, 69.

19 Contreras Castro, Los Peor, 72.

20 Contreras Castro, Los Peor, 73.

21 Véase Contreras Castro, Los Peor, 72-73.
}

186 
El hecho de que se haya traducido este texto costarricense al alemán podría deberse a que probablemente permite confirmar el horizonte de expectativas ${ }^{22}$ de los lectores europeos en relación con la literatura hispanoamericana: el exotismo y la mezcla de lo real con lo mágico. La traducción trata de preservar algo del sabor, de la sonoridad del original a través del abundante uso de préstamos y el empleo de formas de tratamiento del español: por ejemplo, Don Félix, Doña Adelaida, entre otros.

En relación con los préstamos hay que diferenciar aquellos que se usan hoy día en alemán de forma generalizada para referirse a conceptos característicos de la cultura hispana (algunos se han convertido en patrimonio común como, por ejemplo, la siesta), de los préstamos que no le resultan inteligibles al lector medio alemán y que imprimen un cierto carácter exótico al texto traducido: «Dennoch blieb ihnen allen nichts anderes übrig, als in wilder Flucht die Avenida hinunter ihr Heil zu suchen» ${ }^{23}$.

No obstante, el exotismo que se desprende del texto traducido se debe esencialmente al contenido y menos a la forma pues la traducción muestra un elevado grado de neutralización y hasta germanización. Se podría citar, a modo de ejemplo, el hecho de que Jerónimo se refiera a Dios en femenino y que en la narración lo inanimado a menudo se convierta en un ente animado.

La gran difusión de la que goza y ha gozado la lengua y cultura hispana en las últimas décadas (gracias al turismo, la literatura y al interés general por la lengua española) ha contribuido a familiarizar al público alemán con muchos aspectos específicos del mundo hispano y

\footnotetext{
22 Véase Acosta: «El horizonte de expectativas se entiende en una doble dimensión: en primer lugar, como un horizonte literario, esto es, el mundo literario de un determinado momento histórico, que incluye el conocimiento, la formación, el gusto y las convenciones estéticas; además de, en segundo lugar, como un horizonte de la praxis vital u horizonte de expectativas de la vida histórica. Un horizonte de este tipo implica, en consecuencia, un sistema de doble naturaleza: las expectativas codificadas en cada obra del pasado y las expectativas de la experiencia vital del posible lector, que en el acto de recepción incorpora al texto literario», 155.

Contreras Castro, Der Mönch..., 155.
} 
los términos que los designan. La comparación con las condiciones de traducción y recepción, por ejemplo, de Mamita Yunai de Fallas muestra la evolución en este sentido. Es decir que préstamos que en esa época requerían una aclaración - de hecho la traducción de $M a$ mita Yunai va acompañada de un glosario para facilitar la comprensión de los préstamos- hoy día resultan completamente familiares. A un lector alemán ya no le resulta extraño oír hablar de papayas y yucas a diferencia de lo que ocurría hace unas décadas cuando un fruto como el aguacate iba acompañado de un equivalente, Alligatorbirne ${ }^{24}$ (literalmente significa «pera de aligátor»), una correspondencia que ya entonces resultaría extraña para la mayoría de los lectores y que hoy día sería totalmente opaca pues el significante actualmente en uso es «avocado». Por tanto, a pesar de que el texto cuente con un número considerable de préstamos y de elementos híbridos no se percibe un ef ecto de extrañamiento especial.

Entre los ejemplos de neutralización que se pueden citar se encuentra el de "gallopinto», expresión que no se recupera mediante préstamo y tampoco mediante una expresión correspondiente (pues no existe equivalente y tampoco se crea un neologismo) sino que se reexpresa con una traducción explicativa («arroz con judías»). Esta traducción, sin embargo, no permite recuperar todas las características de este plato típico que la Real Academia de la Lengua Española define como localismo costarricense: «Comida hecha a base de arroz y frijoles revueltos y fritos sobrantes del día anterior, que normalmente se come a la hora del desayuno» ${ }^{25}$.

La traducción carece, en ocasiones, de coherencia en la aplicación de las estrategias traductoras. Por ejemplo, en relación con los nombres propios, el traductor opta en general por el préstamo de los

24 M. Schwauss, «Anmerkungen», C. L. Fallas, Die Grüne Hölle (Berlin: Verlag Volk und Welt, 1954) 277: «Aguacate - Alligatorbime, schmackhafte Frucht, die in Form und Farbe unserer Birne ähnelt und hauptsächlich zu Salaten verarbeitet wird".

25 Real Academia Española, Diccionario de la Lengua Española, 21ª ed. (Madrid: Espasa, 2001) 1111 . 
nombres de personas, calles, plazas y lugares, pero a veces no se mantiene ese principio en el texto meta. Así, por ejemplo, el «Mercado Central» no se presta sino que se traduce como großer Markt ${ }^{26}$, es decir Gran Mercado, mientras que el Parque Central aparece o bien en forma de préstamo ${ }^{27}$ o bien traducido como Zentralpark ${ }^{28}$, sin que se sepa muy bien por qué en un caso se hace así y en el otro de forma distinta. Y mientras que el traductor recupera el «Parque Nacional» mediante préstamo, forma una construcción híbrida con el Parque Morazán que aparece como Morazan-Park ${ }^{29}$. Otro ejemplo que muestra la incoherencia en la aplicación de las soluciones traductoras es el caso de los nombres de dos reyes: Alfonso X el Sabio se traduce por Alfonso X. der Weise ${ }^{30}$ mientras que la «Avenida Isabel la Católica» ${ }^{31}$ se mantiene tal cual.

Si bien esta falta de coherencia y homogeneidad en la aplicación de las soluciones traductoras resulta como mínimo desconcertante, lo que realmente resulta problemático en la traducción es el carácter contradictorio producto de querer mantener un cierto sabor localista y que produce un ef ecto exotizante en un texto que, en términos generales, tiende hacia la neutralización e incluso germanización de lo ajeno. El texto meta mantiene todos los nombres de los personajes sin adaptarlos al alemán con una excepción: el nombre del niño que en lugar de llamarlo Polifemo se le pone el equivalente en alemán, Polyphem ${ }^{32}$. Hay casos muy llamativos de germanización, por ejemplo, Handelsblatt como correspondencia de «Diario del Comercio» y grüne Minna como equivalente de «perrera». El empleo de estos términos resulta problemático pues se evocan asociaciones que

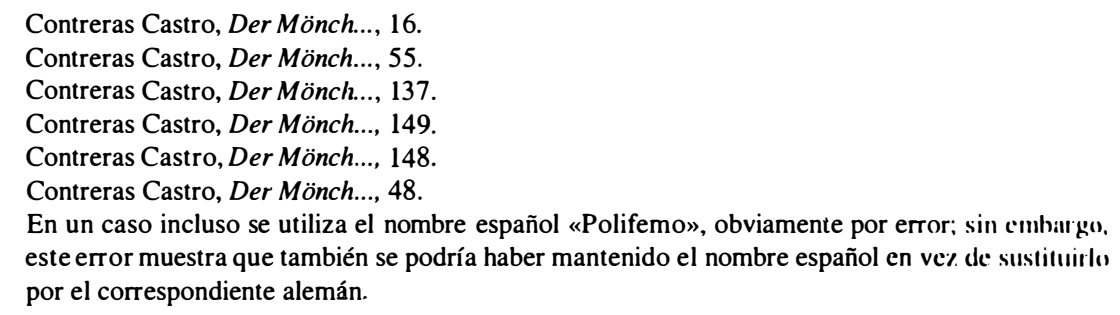

En un caso incluso se utiliza el nombre español «Polifemo», obviamente por error; sin cmharl gu. este error muestra que también se podría haber mantenido el nombre español en vez de sustituirlı, por el correspondiente alemán. 
potencialmente inducen a equívocos: en el primer caso se haría referencia al periódico alemán más importante del ámbito económico y, en el segundo caso, se trataría de un significante de origen y difusión local, aunque sí permitiría recuperar el carácter coloquial que implica el término «perrera».

A los defectos de edición, que se plasman en un incomprensible cúmulo de erratas, se suman omisiones ${ }^{33}$ e imprecisiones que resultan difíciles de asumir. Así, por ejemplo, la «valija de chunches» ${ }^{34}$ se transforma en una maleta abollada ${ }^{35}$ y la frase «a un ropero o a un trinchante» ${ }^{36}$ se convierte en einen unförmigen Schrank zum Beispiel $^{37}$, es decir en un armario desproporcionado, por ejemplo. El traductor seguramente no sabía qué hacer con el «trinchante» - o sea no conocía el real al que apunta este significante-y optó por omitirlo, procedimiento que en ningún caso es aceptable. Tampoco se entiende muy bien por qué reduce el «ropero» en un «armario» y para qué añade la expresión «por ejemplo», que no se encuentra en el texto original.

El elemento mágico - aspecto central pues seguramente explica, al menos en parte, el atractivo de la novela para muchos lectores alemanes - se construye a partir de múltiples personificaciones, una figura retórica a la que el autor recurre asiduamente, así como mediante el relato mitológico-fantástico. Los límites, en ocasiones difuminados, entre lo real y lo fantástico acentúan la impresión mágica que irradia el texto. No es de extrañar que éste logre hacer volar la imaginación del lector con una historia atractiva que le permite experimentar una amalgama inhabitual formada a partir de elementos tan distantes como la mitología e historia greco-latina y su fusión con la compleja realidad de las clases más desfavorecidas de Costa Rica.

33 En la página 179 de la traducción falta un fragmento de la página 213 del texto original.

34 Contreras Castro, Los Peor, 18.

35 Contreras Castro, Der Mönch..., 11.

36 Contreras Castro, Los Peor, 20.

37 Contreras Castro, Der Mönch..., 13. 
De acuerdo con el horizonte de expectativas habría que plantear qué busca el lector alemán en la lectura de una novela hispanoamericana. Es de suponer que lo que más atrae al lector alemán y en general al europeo es el exotismo, la distancia respecto de la propia cultura y el contraste con lo propio. El erotismo es otro aspecto que se suele resaltar al respecto ${ }^{38}$. La novela de Contreras cumple esas expectativas de los lectores, por lo que resulta probable que ése haya sido un factor determinante a la hora de seleccionar este texto para ser traducido y publicado en Alemania. Las referencias al realismo mágico lo vienen a confirmar.

La elección de una novela de carácter más universal para ser traducida al alemán frente a otra que fuese de carácter más localregional confirma la sospecha de que se ha pretendido of recerle al lector alemán un texto que coincida en gran medida con su horizonte de experiencias respecto de la literatura hispanoamericana. Parece razonable pensar que se ha pretendido activar el ef ecto de identificación o Wiedererkennungseffekt a través de un texto al que la crítica relaciona con la tradición del realismo mágico, como muestra la recensión de Klara Obermüller. Desde el punto de vista de la recepción se podría deducir que ahí donde la literatura costarricense se corresponde en buena medida con el horizonte de expectativas de los lectores europeos en relación con la literatura hispanoamericana y va, por tanto, en consonancia con sus prejuicios literarios suscita un mayor interés que si sólo recupera aspectos de carácter más local. La hipótesis de que la elección del presente texto para el mercado alemán pueda deberse a que éste permite a los lectores potenciales confirmar su horizonte de expectativas respecto de la literatura hispanoamericana parece bastante razonable. Esto podría tener repercusiones a medio y largo plazo para la literatura de la región si también lo perciben así los escritores centroamericanos. Sin duda será difícil determinar los

38 La mencionada publicación (ver nota 3) en Alemania en 2002 de una colección de relatos de autores centroamericanos titulada Papayas und Bananen. Erotische und andere Erzählungen aus Zentralamerika. subraya esta idea. Véase W. Mackenbach. 
criterios que utilizan los editores para seleccionar los textos, aunque ya el hecho de elegir unos y no otros permite determinadas conclusiones $^{39}$.

Esta tesis inicial establecida mediante un único caso concreto requerirá una confirmación que esperamos poder of recer con un estudio más exhaustivo que incluya otros textos literarios. De ser cierta nuestra asunción, ese estudio podría aportar datos interesantes sobre las posibilidades de ampliación de la recepción de la literatura costarricense en el extranjero.

39 Acosta: «[...] el surgimiento de la obra literaria, de igual manera que su recepción, tiene lugar dentro de un determinado contexto y horizonte históricos configurados por el autor, los lectores y los lectores críticos. El lector histórico colabora con el acto de recepción de la obra o con los actos de recepción de otras obras en la fijación de un horizonte estético-literario; con sus experiencias de lectura y, dado el caso, con su actividad crítica, está emitiendo juicios [...]. Sólo con el hecho de haberse decidido, dentro de las posibilidades que se le of recen, por la lectura de una u otra obra, y en consecuencia hasta cierto punto haber rechazado otras, ha puesto en evidencia sus apetencias y gustos literarios específicos», 19. 